\title{
Unbelievable Power: The Physics of Nuclear Blast Waves
}

\author{
Matteo Cantiello ${ }^{1}$ \\ ${ }^{1}$ Matteo Cantiello's Blog
}

March 16, 2019

\section{The power of the atom}

At the beginning of the 20th century, major advancements in our understanding of fundamental physics led scientists to the discovery of nuclear energy. An unprecedented amount of power could in principle be released by combining (nuclear fusion) or breaking (nuclear fission) certain atomic species under special conditions. Nuclear fusion in particular was understood to be the process powering the immense luminosity of stars, including our Sun. Nuclear fusion is the energy source illuminating our Universe.

\section{Why so much energy?}

Burning fossil fuels releases chemical energy. This chemical energy is stored in the mild electromagnetic interactions between atoms in a compound. Nuclear energy, on the other hand, comes from the very central regions of the atom. As the name suggests, it is stored in the nuclei, which are kept together by the strong force. The strong force is much stronger than all the other forces, including the electromagnetic one. As a result, nuclear fuel has an energy density about ten million times larger than chemical fuel. If your car was running on nuclear fuel, its gas mileage would be something like hundreds of millions of MPG.

\section{From light to darkness}

The physics revolution that characterized the first three decades of the 20th century and led to the development of quantum mechanics and nuclear physics, was followed by the second World War. In 1942, the United States started a very ambitious project to build a nuclear weapon. The Manhattan Project, led by Robert Oppenheimer and gathering some of the best physicists on the planet, culminated with the successful Trinity experiment in 1945 (Fig.1). The first detonation of a nuclear weapon was the most shocking demonstration of the great power of science and the scientific method. Only less than a month later, two nuclear bombs were dropped over the Japanese cities of Hiroshima and Nagasaki, resulting in the end of WWII and the death of hundreds of thousands of people. The sheer destruction inflicted by the atomic bomb left an indelible mark on humankind's consciousness, formally starting a new era in the history of man. An era of greater responsibility. While no nuclear weapons have been purposely used in war ever since, more than 2000 nuclear tests have been performed after the Trinity, Hiroshima and Nagasaki explosions.

\section{Nuclear weapons testing videos on Youtube}

Between 1945 and 1962 the United States conducted 210 atmospheric nuclear tests. Each event was captured with special cameras, able to record at around 2,400 frames per second. Many declassified films of these tests have been recently retrieved by the Lawrence Livermore National Laboratory (LLNL) and released on Youtube. Below I'll show that is possible to determine the total energy liberated during these tests by estimating the expansion rate of the blast wave. Something that can be done solely from the video of the 


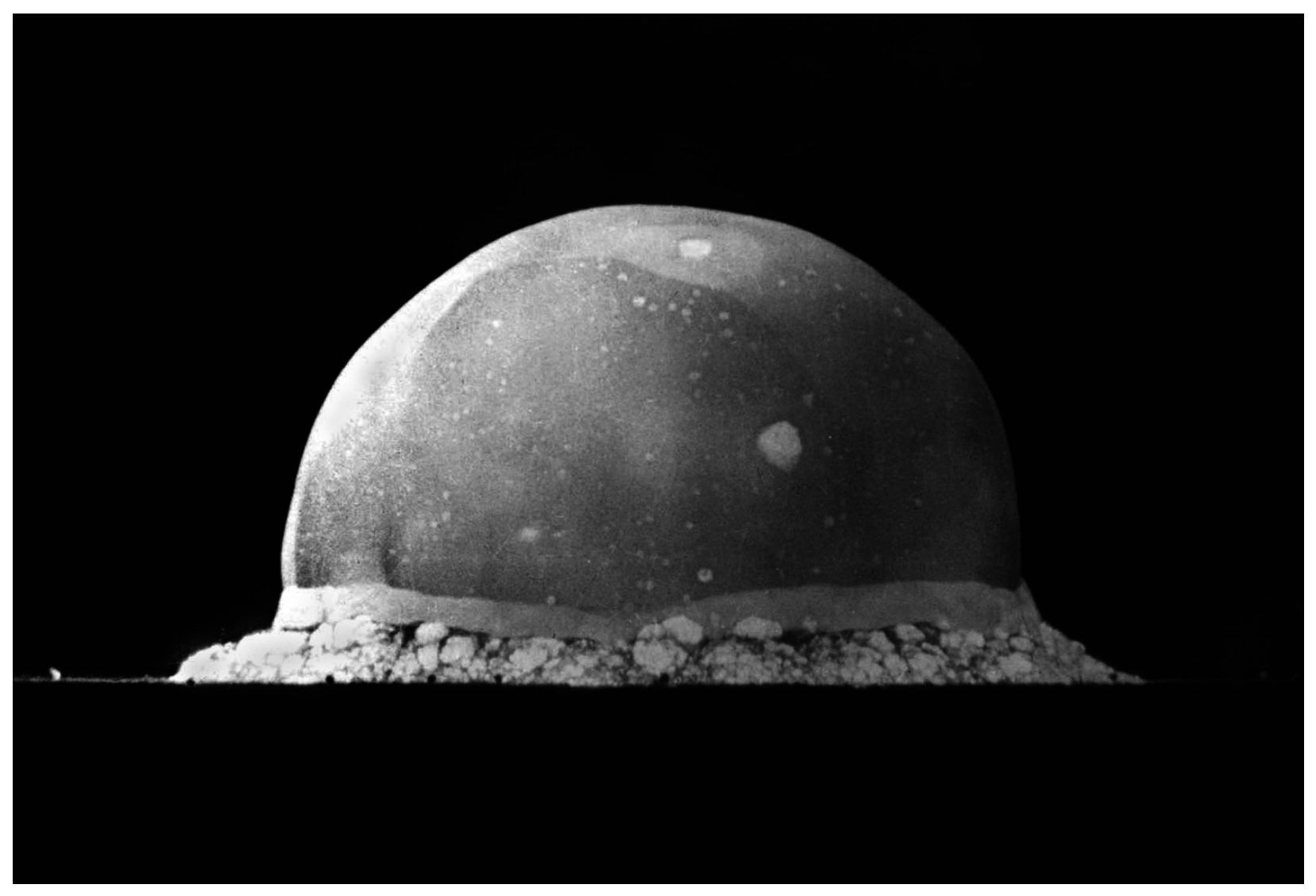

Figure 1: Trinity, the first detonation of a nuclear weapon, was conducted by the US as part of the Manhattan Project on July 161945 in New Mexico. Source: Wikipedia

explosion. This is exactly what two physicists, British Geoffrey I. Taylor and his Russian colleague Leonid Sedov, did after the United States exploded the first atomic bomb in 1945 (the famous Trinity test in New Mexico). Using a few images of the explosions that were shared with the rest of the world by the US army, they determined the total energy released by the new weapon, an information that was highly classified at the time.

\section{"Science: It works, bitches!"}

A nuclear weapon releases, almost instantaneously, a very large amount of energy. This is deposited in a relatively small area, leading to a spherical blast wave expansion. The evolution of this blast wave turns out to be a relatively simple physical problem, since the only important variables of the problem are the total deposited energy $E_{0}$ and the density $\rho_{0}$ of the medium in which the explosion occurs. This means that the radial evolution of the fireball depends only on the energy yield of the weapon and the density of the material in which it exploded (in this case air). Conversely, if one can measure the expansion rate of the fireball in a medium of known density, one can retrieve the energy of the explosion. I tried reproducing what Sedov and Taylor did in 1945 with the Trinity test, using one of the newly released footage from the Tesla test, part of operation Teapot. This particular bomb was exploded on the 1st of March 1955 in the Nevada test site, some 65 miles north west of Las Vegas. In Figure 2 six frames extracted from the youtube video show the expansion of the blast wave. Below I re-derived the Sedov-Taylor solution, which I used to fit the data and estimate the total energy of the blast in Figure 3. The data and the code I used is accessible 
through the data icon in that figure. The best fit is obtained for an energy of 4.3 kilotons, which given the approximations and the very rudimental way I used to determine the location of the blast wave radius, is in very good agreement with the value declared by LLNL (7 kilotons). Science works! You can try doing this with some of the other videos, and check if the declared energy of the weapon matches what you find. For more details on the Taylor-Sedov solution see Taylor (1950); SEDOV (1959).

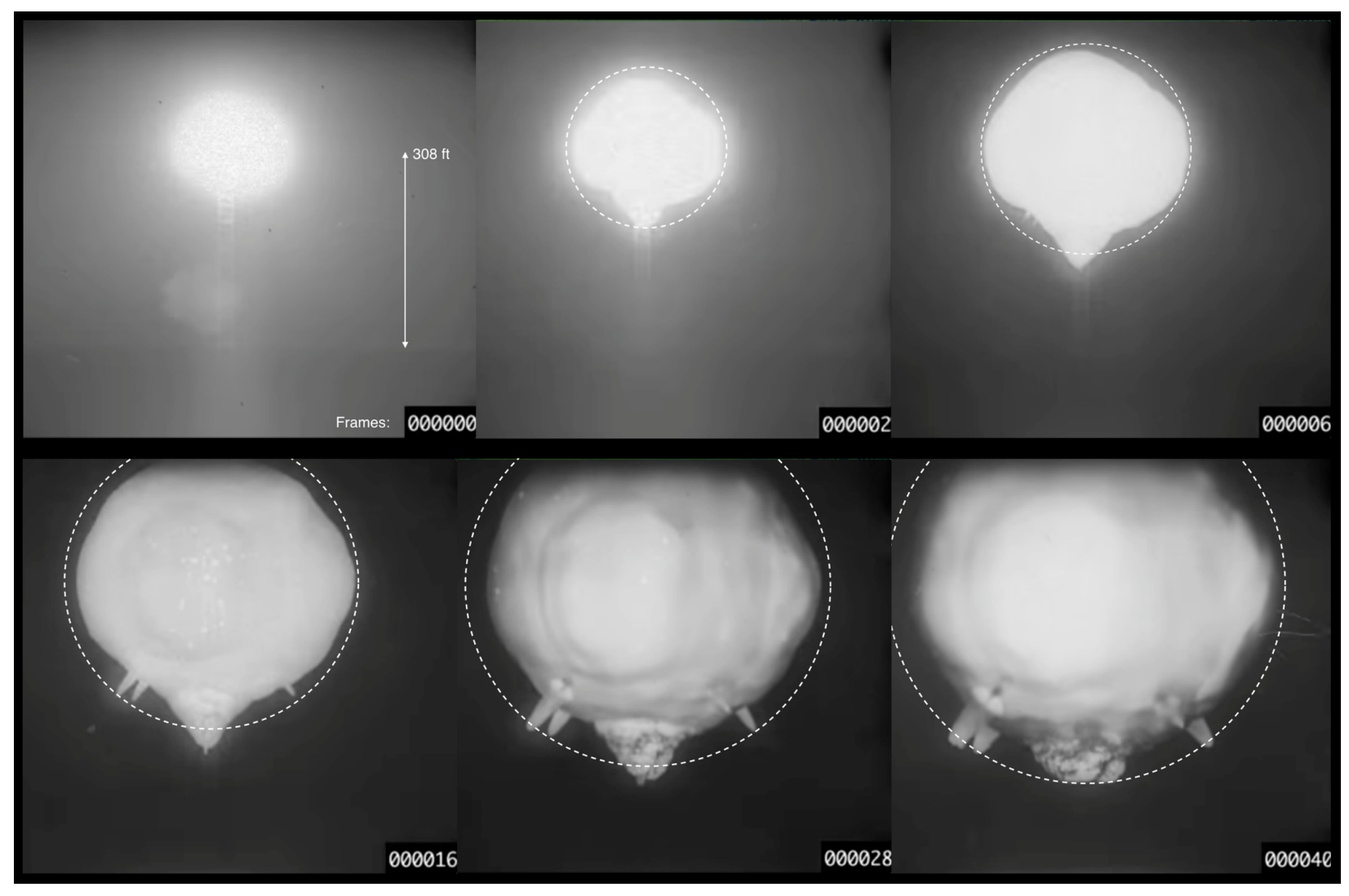

Figure 2: Frames sequence extracted from the recently declassified video of the "Tesla" nuclear test, part of operation Teapot (Nevada, 1955). The weapon was placed on top of a $308 \mathrm{ft}$ tall tower (visible in the top-left frame). The high-speed camera was recording about 2,400 frames per second. The 'spikes' visible on the lower part of the expanding fireball are due to mooring cables being vaporized by the radiative precursor (rope trick effect), debris of the weapon's casing are visible in frames 16 and 28. We estimated the approximate position of the blast wave from the radius of the fireball at different times (overlaid dashed circles). Watch the original video here. Credits: LLNL

\section{Sedov-Taylor Solution}

We want to determine the evolution with time of the radius of the spherical blastwave. We assume that the explosion is releasing almost instantaneously a very large amount of energy in a medium with constant density. In this case the only variables of the problem are the initial energy of the explosion $E_{0}$ and the initial density $\rho_{0}$ of the medium. These two quantities have dimensions $\left[E_{0}\right]=M \cdot L^{2} \cdot t^{-2}$ and $\left[\rho_{0}\right]=M \cdot L^{-3}$, where $M, L, t$ represent dimensions of mass, length and time, respectively. We can then find the evolution equation of the fireball noticing that there is only one way to combine $E_{0}$ and $\rho_{0}$ in order to obtain a quantity 
with dimensions of a length:

$$
\frac{\left[E_{0}\right]}{\left[\rho_{0}\right]} \propto \frac{L^{2} \cdot t^{-2}}{L^{-3}}=L^{5} \cdot t^{-2} \Rightarrow L \propto\left(\frac{E_{0}}{\rho_{0}}\right)^{1 / 5} t^{2 / 5}
$$

The equation that determines the evolution of the fireball radius is therefore:

$$
R(t)=A\left(\frac{E_{0}}{\rho_{0}}\right)^{1 / 5} t^{2 / 5}
$$

where $A$ is a dimensionless constant of order unity (see the Appendix).

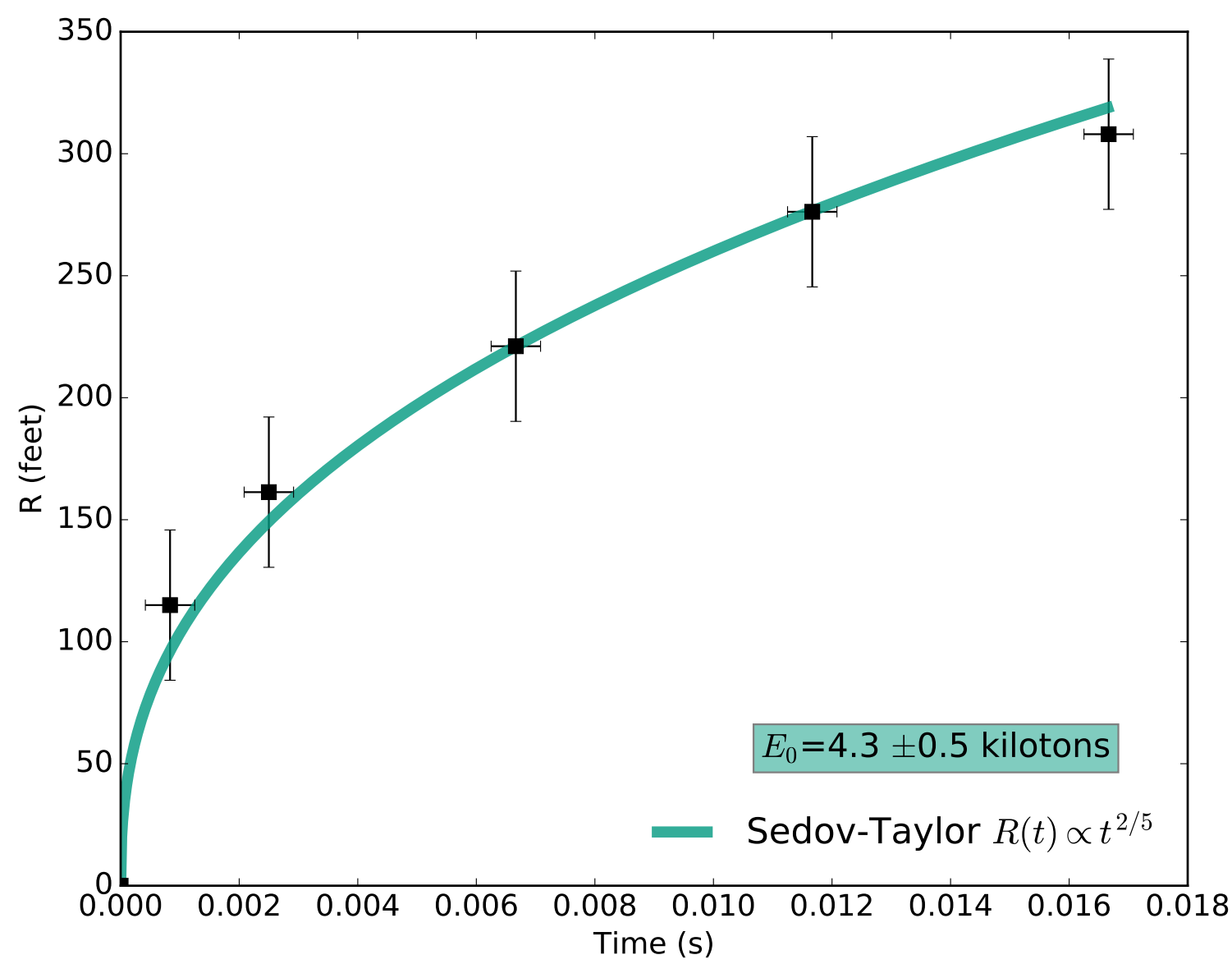

Figure 3: Since the air density is known, the Sedov-Taylor solution has one single parameter (the initial blast energy $E_{0}$ ) which we determined fitting the observed data obtained from the recently declassified video posted on youtube. The best fit is obtained for $E_{0}=4.3$ kilotons, which is the right order of magnitude when compared to the energy declared by LNLL for this experiment (7 kilotons). Python code used to generate this figure. 


\section{Appendix}

To constrain the dimensionless factor we can impose conservation of total energy. We assume that all the energy is going into kinetic energy of the fireball. Note that this is not completely true, as some energy has to go into radiation as well (the explosion is very luminous, some air is ionized etc). We will also assume that all the mass swept up by the expanding fireball is confined in a shell of width $\Delta R$ (with $\Delta R / R \ll 1$ ) moving with a velocity $v(t)=\dot{R}(t)$. The total energy is then:

$$
E=\frac{1}{2} m v^{2}=\frac{1}{2}\left(\frac{4}{3} \pi R^{3} \rho_{0}\right) \dot{R}^{2}
$$

We can calculate $\dot{R}$ deriving respect to time Eq. 1 :

$$
\dot{R}(t)=A \frac{2}{5}\left(\frac{E_{0}}{\rho_{0}}\right)^{1 / 5} t^{-3 / 5}
$$

Imposing energy conservation $\left(E=E_{0}\right)$ we obtain $A=\left(\frac{75}{8 \pi}\right)^{1 / 5} \simeq 1.24$.

A more detailed calculation reveals $A \simeq 1.16$, see e.g. Chapter 17 of Shu (1992).

\section{Acknowledgements}

I want to thank Daniel Lecoanet for some fun and related discussion we had in Princeton, and Steve N. Shore for being the first one to introduce me to the story of the calculation of the Trinity test energy by Sedov and Taylor. 


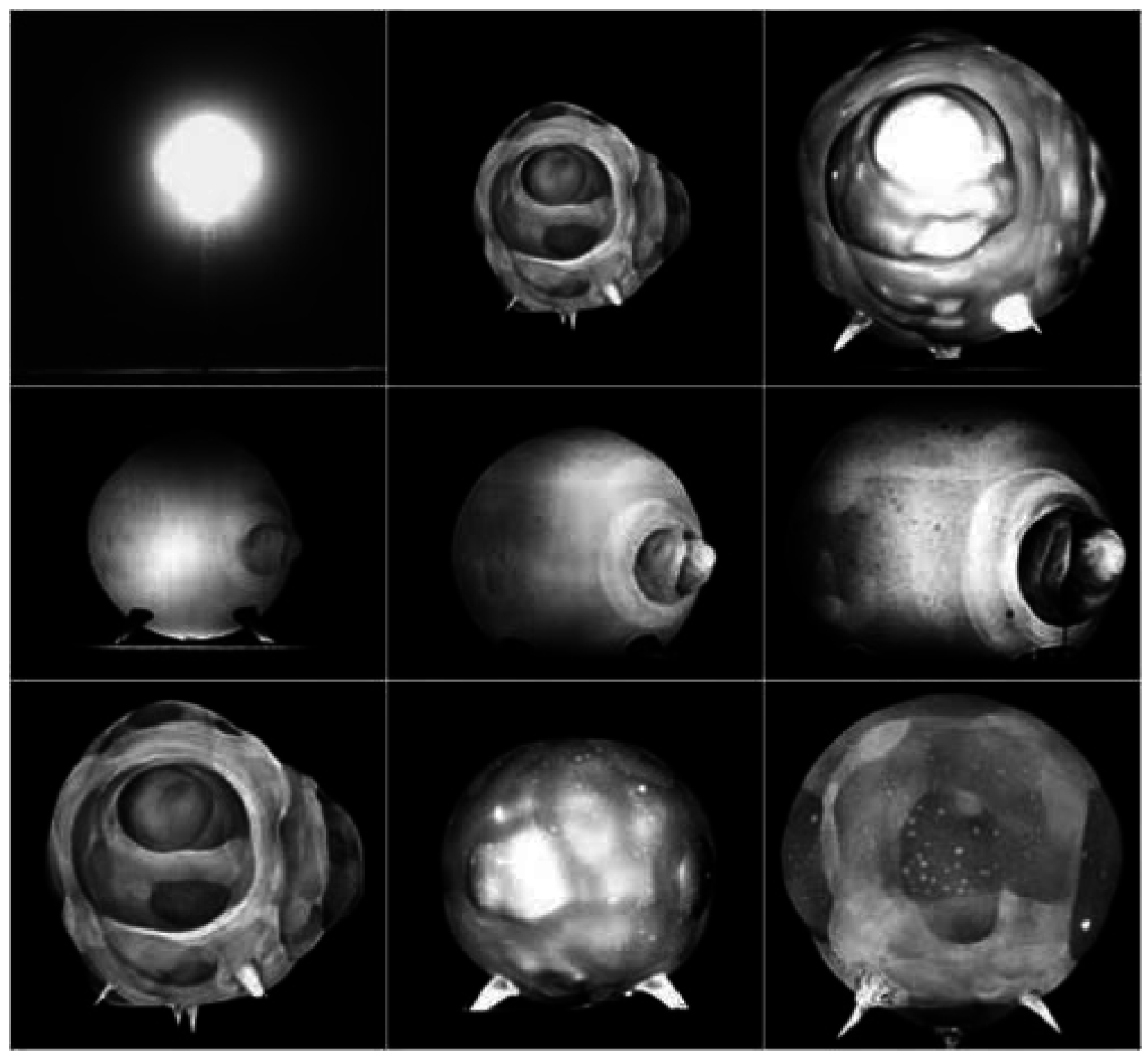

Figure 4: Rapatronic shots of the early phase of nuclear weapon detonations. The magnetic shutter in the rapatronic allows to record a still image with an exposure time as brief as 10 nanoseconds (Source: Photographic History Collection at Smithsonian's National Museum of American History) 


\section{References}

L.I. SEDOV. General Dimensional Theory. In Similarity and Dimensional Methods in Mechanics, pages 123. Elsevier, 1959. doi: 10.1016/b978-1-4832-0088-0.50008-6. URL https://doi.org/10.1016\%2Fb9781-4832-0088-0.50008-6.

F. H. Shu. The physics of astrophysics. Volume II: Gas dynamics. 1992.

G. Taylor. The Formation of a Blast Wave by a Very Intense Explosion. I. Theoretical Discussion. Proceedings of the Royal Society A: Mathematical Physical and Engineering Sciences, 201(1065):159-174, mar 1950. doi: 10.1098/rspa.1950.0049. URL https://doi.org/10.1098\%2Frspa.1950.0049. 